\title{
Simulation of contact heat and mass transfer in spray and bubbling apparatuses
}

\author{
Michael Shilyaev, Helen Khromova, and Alexander Tolstykh ${ }^{\mathrm{a}}$ \\ Tomsk State University of Architecture and Building, 634003 Tomsk, Russia
}

\begin{abstract}
The results of numerical implementation of mathematical models of heat and mass transfer and complex condensation-absorption dust- and gas cleaning of the vapor-gas flows are compared with the known empirical data for HSS, VS, FA, and CBA on heat and mass transfer characteristics and efficiency of dust catching.
\end{abstract}

The spray (the irrigation chambers of air conditioners (AC), hollow spray scrubbers (HSS), Venturi scrubbers (VS), and bubbling (foam (FA), centrifugal-bubbling (CBA)) apparatuses are widely used in industry for heat-moisture gas processing, their cleaning from dust and harmful gas components. However, until now the design of theses apparatuses was performed on the basis of empirical data only, restricted by the range of physical, geometrical and operation parameters of their obtaining [1, 2]. This circumstance excludes optimization of this equipment and its sizes by the efficiency of processes, occurring there, and effectiveness of its operation.

At the Department of heating and ventilation of TSUAB the authors of this equipment have developed the mathematical models of heat and mass transfer and complex condensation-absorption dust- and gas cleaning of the vapor-gas flows. The main ideas of these models are presented in [3]. In the current paper we have extended these ideas for catching fine polyfractional dust and high temperatures of processed gases.

The results of numerical implementation of these models are compared with the known empirical data for HSS, VS, FA, and CBA on heat and mass transfer characteristics and efficiency of dust catching.

Thus, results of comparative calculations by the mathematical model with two-sided irrigation (forward flow and counterflow) of heat transfer in irrigation chamber of AC, suggested by the authors, and the empirical VNIIConditioner method at different, but close irrigation coefficients $q$, corresponding with each other, are presented in Table 1.

*Air and water parameters at the inlet of the irrigation chamber of AC: temperature, moisture content and air velocity are as follows: $T_{00}=298.9 \mathrm{~K}, d_{0}=10.4 \mathrm{~g} / \mathrm{kg}$ dry air, $U_{0}=2.67 \mathrm{~m} / \mathrm{s}, V_{d 0}=15.26 \mathrm{~m} / \mathrm{s}$, $\delta_{d 0}=457 \mu \mathrm{m}$. Here $q$ is irrigation coefficient, $\Theta_{0}$ and $V_{d 0}$ are temperature and velocity of droplets at the inlet, $T_{1}, d_{1}, \Theta_{1}$ are air temperature, moisture content and droplet temperature at the chamber outlet.

Calculation results on the efficiency of soot catching from cracking gases through condensation in HSS are shown in Fig. 1. According to the figure, the experimental value of efficiency under the conditions of industrial scrubber testing coincides well with calculations. It can be also seen from this

\footnotetext{
${ }^{a}$ Corresponding author: shmi@mail .tomsknet.ru
}

This is an Open Access article distributed under the terms of the Creative Commons Attribution License 4.0, which permits unrestricted use, distribution, and reproduction in any medium, provided the original work is properly cited. 
Table 1. Comparison of operation parameters of AC irrigation chamber.

\begin{tabular}{|c|c|c|c|c|c|c|}
\hline Parameters & VNIIConditioner method & \multicolumn{5}{|c|}{ Heat and mass transfer model } \\
\hline$q, 1 / \mathrm{m}^{3}$ & 2.33 & 2.33 & 2.1 & 1.95 & 1.8 & 1.7 \\
\hline$\Theta_{0}, \mathrm{~K}$ & 277.86 & 277.86 & 277.0 & 276.0 & 275.5 & 275.0 \\
\hline$T_{1}, \mathrm{~K}$ & 284.3 & 285.05 & 285.18 & 285.08 & 285.36 & 285.49 \\
\hline$d_{1}, \mathrm{~g} / \mathrm{kg}$ & 7.8 & 7.3 & 7.3 & 7.2 & 7.2 & 7.2 \\
\hline$\Theta_{1}, \mathrm{~K}$ & 280.54 & 280.12 & 279.5 & 278.74 & 278.42 & 278.07 \\
\hline
\end{tabular}

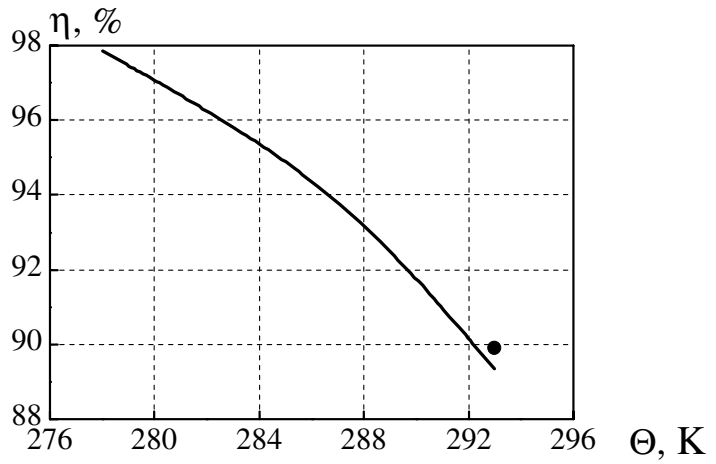

Figure 1. Results of calculations by the model for HSS: $H=12.75 \mathrm{~m}, \tilde{q}=7.1 \cdot 10^{3} \mathrm{~m}^{3} / \mathrm{m}^{3}, \delta_{d 0}=7 \cdot 10^{-4} \mathrm{~m}$, $V_{d 0}=24.5 \mathrm{~m} / \mathrm{s}, T_{00}=443 \mathrm{~K}, U_{0}=0.25 \mathrm{~m} / \mathrm{s}, d_{0}=0.93 \mathrm{~kg} / \mathrm{kg}$ dry air, $\delta_{p 0}=10^{-7}, c_{p 0}=1.72 \mathrm{~g} / \mathrm{m}^{3}$.

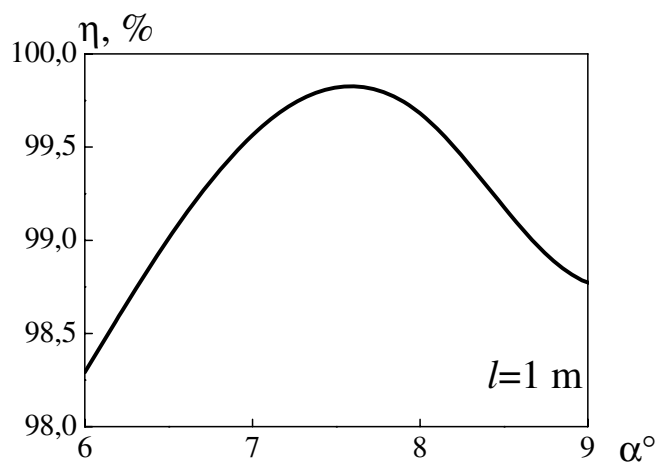

Figure 2. The effect of opening angle on efficiency of dust catching: $V_{0}=5 \mathrm{~m} / \mathrm{s}, \Theta_{0}=293 \mathrm{~K}, \rho_{m}=10^{3} \mathrm{~kg} / \mathrm{m}^{3}$, $\tilde{q}=10^{-3} \mathrm{~m}^{3} / \mathrm{m}^{3}, U_{0}=80 \mathrm{~m} / \mathrm{s}, T_{00}=333 \mathrm{~K}, c_{p 0}=1 \mathrm{~g} / \mathrm{m}^{3}, d_{0}=0.5 \mathrm{~kg} / \mathrm{kg}$ dry air, $\delta_{p 0}=10^{-6} \mathrm{~m}$.

figure that efficiency can be significantly improved due to a decrease in the temperature of irrigating water. It also follows that the same experimental value of efficiency can be achieved in a scrubber with the height of about 3 times lower at water temperature of $+5^{\circ} \mathrm{C}$ instead of experimental $+20^{\circ} \mathrm{C}$.

Results of calculation of efficiency of fine dust catching through the condensation method in VS are shown in Fig. 2 depending on opening angle $\alpha$ of its diffuser. It is obvious that the optimal value of $\alpha$ in this case is $7.7^{\circ}$; the value of $\alpha$ recommended from the experimental data is $6-7^{\circ}$ [2].

Calculation results of fractional efficiency of dust catching in VS under the isothermal conditions are shown in Fig. 3 in comparison with the known generalized experimental dependence

$$
\eta_{\delta}=1-\mathrm{e}^{-K q \sqrt{\mathbf{S t k}}},
$$




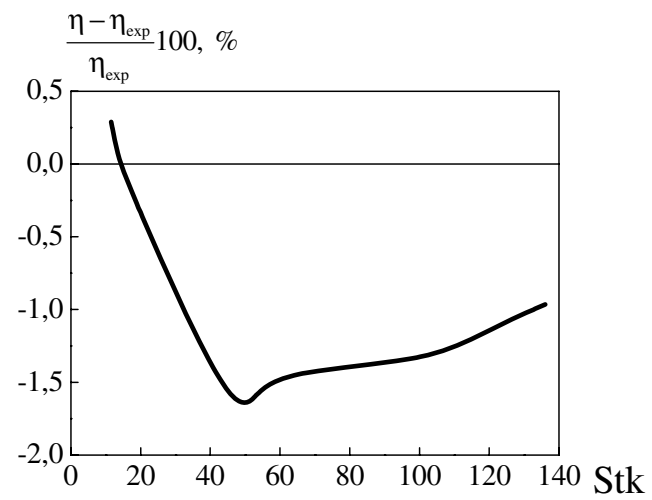

Figure 3. Comparison of the model with experimental data under the isothermal conditions.

$a$

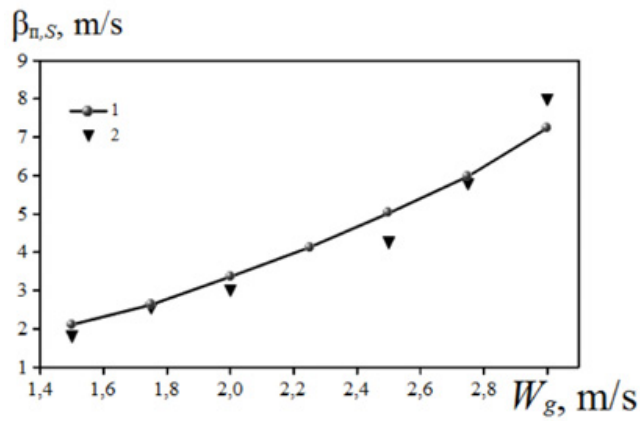

b

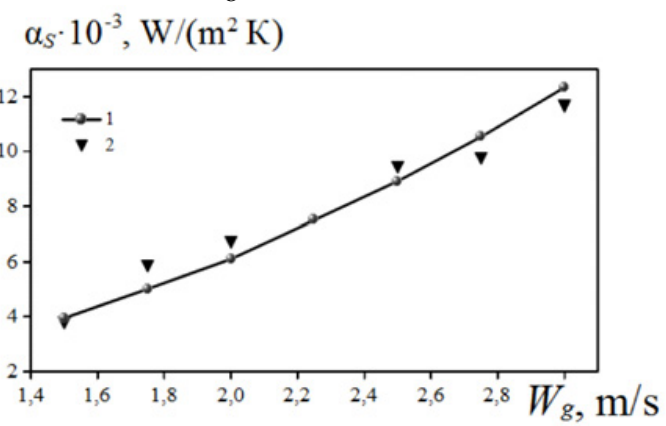

Figure 4. Dependence of mass transfer coefficients $\beta_{\mathrm{n}, S}(a)$ and mass transfer coefficients $\alpha_{S}(b)$ on superficial velocity of the vapor-gas mixture $W_{g}$ in CBA (cylindrical bubble): 1 - calculation by the model; 2 - experimental data: $t_{g 0}=(62-44)^{\circ} \mathrm{C}, d_{0}=0.0093 \mathrm{~kg} / \mathrm{kg}$ dry air.

where $K=1.5$ is geometrical parameter, Stk $=\frac{\rho_{m} \delta^{2}}{18 \mu} \frac{V_{c}}{\delta_{d}}, \rho_{m}$ is density of particles, $\mathrm{d}$ is diameter of particles, $\mu$ is dynamic viscosity of gas, $V_{c}=U_{0}-V_{d 0}, U_{0}, V_{d 0}$ is velocity of gas and droplets in the neck of Venturi tube, $\delta_{d}$ is the size of droplets calculated by Hukiyama-Tanasawa formula. According to the figure, the difference between experimental $\eta_{\exp }$ and calculated $\eta$ efficiencies does not exceed $2 \%$.

The absorption processes of $\mathrm{CO}_{2}, \mathrm{CH}_{4}, \mathrm{H}_{2} \mathrm{~S}$, and $\mathrm{SO}_{2}$ extraction from air with and without dust on water droplets in HSS and VS were calculated and agreed with experimental data of [1] within difference of $6-13 \%$.

The mathematical model of heat and mass transfer in FA and CBA was developed for temperaturestabilized and not-temperature-stabilized liquid on gas-distributing grates. Calculation results are compared with experimental data on heat and mass transfer coefficients and presented in Fig. 4.

Results of comparison of calculation and experimental data for the heat and mass transfer processes and dust catching in HSS, VS, FA, and CBA prove the validity of suggested models and possibility of their efficient application for engineering calculations and optimization of this equipment operation. 


\section{References}

[1] V.M. Ramm, Absorption of Gases (Khimiya, Moscow, 1976)

[2] A.A. Rusanov, Hand-Book on Dust and Ash Catching (Energia, Moscow, 1975)

[3] M.I. Shilyaev, E.M. Khromova, A.V. Tolstykh, Contact heat and mass transfer in spray and bubbling apparatuses. Simulation and optimization of heat and mass transfer and absorptioncondensation dust and gas cleaning (LAP LAMBERT Academic Publishing, Germany, 2012) 